\title{
Pharmaprix ou Pharmabraconnage?
}

Diffusion anticipée sur www.cmaj.ca le 30 novembre 2007. Revisé le 4 janvier, 2008.

$S$ hoppers Drug Mart (Pharmaprix au Québec) est une institution canadienne bien connue. Cette chaîne de pharmacies, la plus importante au Canada, compte Iooo points de vente partout au pays. Dans beaucoup de communautés canadiennes, les franchises locales de l'entreprise sont solidement dotées en personnel et dispensent des soins de qualité aux patients.

En Afrique du Sud, toutefois, la même entreprise risque de contribuer à une catastrophe en santé publique. En 2005, 2006 et 2 fois en 2007, elle y a dépêché des recruteurs chargés de trouver des pharmaciens dans un continent qui en a déjà trop peu pour répondre à ses besoins. Shoppers Drug Mart promet des salaires dans les 6 chiffres et de l'aide pour lancer une brillante carrière au Canada. La carotte, et la main bienfaisante qui la tient, sont énormes.

La plupart des Canadiens ont probablement déjà été soignés par un médecin, une infirmière ou un pharmacien venant d'Afrique du Sud. La migration est bonne pour le Canada parce que les travailleurs de ce pays sont en général d'excellente qualité. Elle peut toutefois se révéler catastrophique pour l'Afrique du Sud, car elle coïncide avec une épidémie de sida : on estime que I9 \% des adultes sud-africains sont infectés par le VIH. Les journaux de l'Afrique du Sud signalent que des patients doivent attendre plusieurs jours simplement pour faire remplir une ordonnance ${ }^{1}$. Les provinces les plus durement frappées, comme le KwaZulu-Natal, ont besoin de centaines de nouveaux pharmaciens pour distribuer des médicaments contre le sida, mais récemment, les trois quarts des postes de pharmacien offerts par l'État étaient toujours vacants ${ }^{2}$.

Étant donné l'histoire extraordinaire de l'Afrique du Sud, ces difficultés ont de nombreuses causes. La migration incessante de professionnels de la santé est une cause majeure. Les journaux médicaux du pays publient des masses d'offres d'emploi à l'étranger - il y en a eu des milliers au cours des dernières années seulement ${ }^{3}$. Tout travailleur de la santé compétent peut trouver un débouché et émigrer. Beaucoup le font pour des raisons familiales ou professionnelles légitimes, comme ils en ont indéniablement le droit.

L'attrait passif d'une offre d'emploi fait toutefois contraste avec une pratique différente, celle du recrutement actif, ciblé et systématique de pharmaciens employables et d'autres professionnels de la santé - pratique mieux connue sous le nom de braconnage - du bassin de talents de l'Afrique du Sud.

C'est cette forme de braconnage que pratique Shoppers Drug Mart. Une ou 2 fois par année, le siège social de l'entreprise envoie des missions de recrutement en Afrique du Sud pour attirer et interviewer des candidats. On promet aux candidats «un revenu annuel minimum garanti de Ioo ooo \$» - qui représente une augmentation énorme comparativement au salaire typique en Afrique du Sud ${ }^{4}$. Pour aider les intéressés à quitter facilement la maison, Shoppers Drug Mart offre même les services de son cabinet d'avocats qui «aidera (...) à présenter une demande d'immigration ${ }^{4} »$. L'appareil de recrutement de l'entreprise vise les pharmaciens actifs, les étudiants en pharmacie et même les professeurs de pharmacie (comme l'un d'entre nous, R.B.W.) sans lesquels l'Afrique du Sud ne peut lutter contre sa pénurie de pharmaciens.

Shoppers Drug Mart n'a pas honte de ce recrutement : elle en est même fière. Elle se vante sur son site web d'aider depuis longtemps des pharmaciens d'autres pays à lancer une nouvelle carrière au Canada ${ }^{4}$.

Or, ce comportement n'est pas simplement maladroit : il est contraire à l'éthique. Il s'agit en fait d'une entreprise canadienne qui profite des contribuables de l'Afrique du Sud et de son système appauvri d'enseignement supérieur - cela équivaut à faire de l'aide étrangère à l'envers. Si Shoppers Drug Mart a besoin de travailleurs, elle ne devrait pas les braconner : elle devrait plutôt les former en payant des bourses d'études à des écoles de pharmacie du Canada et de l'Afrique du Sud. Shoppers Drug Mart verse un généreux dividende aux actionnaires et prévoit investir 400 millions de dollars en capital immobilisé pour étendre son réseau de magasins cette année ${ }^{5}$. Elle n'est pas trop pauvre pour investir dans la formation des travailleurs en pharmacie qui constituent son capital humain.

Un système réfléchi et respectueux de l'éthique offrirait une solution gagnante pour tous : les écoles de pharmacie augmenteraient le nombre de candidats qu'elles acceptent grâce à des bourses d'études subventionnées, Shoppers Drug Mart y trouverait les pharmaciens bien formés dont elle a besoin, et les patients de l'Afrique du Sud qui souffrent depuis longtemps auraient plus de chances d'avoir accès aux pharmaciens dont ils ont besoin.

Qu'est-ce qui arrête Shoppers Drug Mart? Les bourses d'études coûtent de l'argent et la chaîne de pharmacies opère dans une industrie où le braconnage constitue une norme honteuse. L'Association canadienne des chaînes de pharmacies ne décourage pas le braconnage; au contraire, elle «appuie des mesures qui accélèrent la capacité des diplômés de facultés de pharmacie étrangères à immigrer au Canada ${ }^{6}$.

Il ne fait guère de doute que Shoppers Drug Mart n'est pas la seule chaîne de pharmacies à pratiquer le braconnage, mais comme elle est la plus grande entreprise de sa catégorie, elle se trouve dans une situation unique pour hausser la barre souterraine de l'éthique de l'industrie face à cette pratique. Depuis la dernière décennie, des pays, des associations professionnelles et le Commonwealth ont adopté des codes de conduite qui désapprouvent en général le braconnage dans les professions de la santét. Ces codes lient moralement, sinon légalement, et les entreprises qui se veulent socialement responsables doivent les observer. Les braconniers 
méritent de perdre des clients. Les ministres de la Santé du Commonwealth recommandent, par exemple, que les gouvernements envisagent "d'employer seulement les agences [privées] de recrutement qui se conforment au Code [du Commonwealth] et de transiger seulement avec elles $»^{8}$.

La mission de recrutement de Shoppers Drug Mart s'est rendu à Pretoria, Johannesburg, Durban et Le Cap. Le JAMC croit que Shoppers Drug Mart devrait annuler les expéditions de braconnage à venir et s'engager à mettre en œuvre un moyen basé sur la formation pour répondre à ses besoins en effectifs - solution dont elle pourrait être la pionnière et que d'autres membres de l'industrie des chaînes de pharmacies pourraient ensuite adopter à leur tour. Si Shoppers Drug Mart n'agit pas, le JAMC croit aussi que les gouvernements, les hôpitaux et tous les Canadiens devraient faire preuve de solidarité pour l'Afrique du Sud et aller acheter ailleurs.

\section{Addenda}

I) Suite à la parution anticipée en ligne de cet éditorial le 26 novembre 2007 , un membre de l'équipe de rédaction de l'éditorial du JAMC a communiqué avec Pharmaprix (Shoppers Drug Mart) le 27 novembre 2007 et a invité l'entreprise à présenter une lettre au journal pour exposer sa position. Au cours de cette discussion, Pharmaprix a confirmé qu'elle examinait ses pratiques de recrutement, mais n'a pas confirmé l'annulation de ses activités de recrutement en Afrique du Sud.

2) Pharmaprix semble donc avoir poursuivi ses activités de recrutement en Afrique du Sud selon un rapport publié en ligne par The Globe and Mail le 3 décembre9.

3) Comme le souligne deux articles de la section Actualités du présent numéro, le braconnage de professionnels entre pays est une pratique répandue (JAMC 2008;178(3):269-7I).

4) Une telle pratique est particulièrement inquiétante lorsque des pays industrialisés cherchent systématiquement à combler leurs besoins en ressources humaines en recrutant des professionnels auprès de pays dont les besoins sont infiniment plus grands et où les pénuries beaucoup plus graves.

5) Pour résoudre les pénuries de professionnels de la santé dans le monde non industrialisé, il faut faire bien plus que sim- plement interdire le braconnage. Une telle interdiction constitue toutefois un élément essentiel d'une approche intégrée.

\section{Amir Attaran LLB DPhil}

Titulaire de la Chaire de recherche du Canada en droit, santé des populations et politique du développement mondial

Université d'Ottawa

Ottawa (Ont.)

Roderick B. Walker PhD

Doyen désigné, Faculté de pharmacie

Université Rhodes

Grahamstown, Afrique du Sud

Avec l'équipe de rédaction de l'éditorial (Paul C. Hébert MD MHSc, Matthew B. Stanbrook MD PhD, Ken Flegel MDCM MSc et Noni MacDonald MD MSc)

\section{RÉFÉRENCES}

I. Ryan M. Patients in long wait for medicine. Sunday Tribune [Afrique du Sud] 7 janvier 2007.

2. Ryan M. State pharmacies' staffing crisis. Sunday Tribune [Afrique du Sud] 28 janvier 2007.

3. Rogerson CM. Medical recruits: the temptation of South African healthcare professionals [policy paper]. Southern African Migration Project. Kingston (ON): The Project; 2007. Disponible à : www.queensu.ca/samp/sampresources/samppublications/ (consulté le 23 novembre 2007).

4. Shoppers Drug Mart. [International recruitment websites] 2007. Disponible à : www.shoppersdrugmart.ca/french/careers/international/index.html et http://www.shoppersdrugmart.ca/english/careers/international/africa.html et www.shoppersdrugmart.ca/french/careers/international/visas_permits/index.html et www.shoppersdrugmart.ca/french/careers/international/students_interns/index html (consulté le 23 novembre 2007)

5. Shoppers Drug Mart. [Rapport annuel] 2006. Disponible : www.shoppersdrugmart .ca/french/corporate_information/investor_relations/financial_information/annual _report/full_report/SDM_06\%20French\%2ofull.pdf(consultéle 23 novembre 2007).

6. Association canadienne des chaînes de pharmacies. Lettre à Jodi White, Forum des politiques publiques. 24 juin 2005. Disponible à : www.cacds.com/files ICACDS\%2oResponse\%20to\%20PPF\%20Role\%20of\%2oGovernments\%20June\% 202005.pdf (consulté le 23 novembre 2007).

7. Pagett C, Padarath A. A review of codes and protocols for the migration of health workers. Causeway, Harare (Zimbabwe): The Regional Network for Equity in Health in east and southern Africa (EQUINET); 2007. Disponible à : www.equinetafrica .org/bibl/docs/Dis5oHRpagett.pdf (consulté le 23 novembre 2007)

8. Ministres de la Santé du Commonwealth. Companion document to the Commonwealth Code of Practice for the International Recruitment of Health Workers. Londres : Le Secrétariat du Commonwealth; 2003. Disponible : www.thecommonwealth.org /shared_asp_files/uploadedfiles/\%7B7F307830-IFA4-44A3-9EA5-9CC4055D7D4B $\% 7$ D_CompanionDocument.pdf (consulté le 23 novembre 2007).

9. Nolen S. Canadian firm poaches pharmacists from South Africa. The Globe and Mail. 3 décembre 2007. Available: www.shns.com/shns/scrippsnews/index.cfm ?action=detail\&pk=SAFRICADRUGGISTS-I2-03-07 (consulté le 4 janvier 2008). 